\title{
OGTT 2-hour serum C-peptide index as a predictor of post- transplant diabetes mellitus in kidney transplant recipients
}

\author{
Xingqiang Lai, Lei Zhang, Jiali Fang, Guanghui Li, Lu Xu, Junjie Ma, Yunyi Xiong, Luhao Liu, \\ Zheng Chen
}

Organ Transplant Center, The Second Affiliated Hospital of Guangzhou Medical University, Guangzhou 510260, China

Contributions: (I) Conception and design: X Lai, Z Chen; (II) Administrative support: Z Chen; (III) Provision of study materials or patients: L Zhang, J Fang, G Li, J Ma, Z Chen; (IV) Collection and assembly of data: X Lai, L Xu, Y Xiong, L Liu; (V) Data analysis and interpretation: X Lai, L Zhang, J Ma; (VI) Manuscript writing: All authors; (VII) Final approval of manuscript: All authors.

Correspondence to: Zheng Chen. Organ Transplant Center, The Second Affiliated Hospital of Guangzhou Medical University, No. 250 Changgang East Road, Haizhu District, Guangzhou 510260, China. Email: docchenzheng@163.com.

\begin{abstract}
Background: The high incidence of post-transplant diabetes mellitus (PTDM) necessitates the identification of new factors to explain its pathogenesis. This study aimed to clarify the association between the C-peptide index (CPI) and PTDM.
\end{abstract}

Methods: A total of 290 non-diabetic kidney transplant patients were analyzed. All subjects underwent a $75 \mathrm{~g}$ oral glucose tolerance test (OGTT). Plasma glucose concentrations, serum C-peptide levels, hemoglobin A1c (HbA1c), and other biochemical indicators were measured. CPI was calculated as the ratio of serum C-peptide to plasma glucose.

Results: Among the 290 patients, 36 (12.4\%) developed PTDM at the end of 1 year. Patients with PTDM had older age $(\mathrm{P}<0.001)$, higher levels of body mass index (BMI) $(\mathrm{P}=0.004)$ and HbA1c $(\mathrm{P}=0.001)$, a higher proportion of deceased donors $(\mathrm{P}=0.045)$, and lower levels of $2 \mathrm{~h}-\mathrm{CPI}(\mathrm{P}=0.02)$ than those without PTDM. The OGTT 2 h-CPI was positively correlated with BMI, HbAlc, type of calcineurin inhibitor, albumin, and triglyceride. Multivariate logistic regression and Cox hazard model analysis showed that pre-transplant OGTT $2 \mathrm{~h}-\mathrm{CPI}$ was an independent predictor for the development of PTDM, together with age, BMI, and HbA1c.

Conclusions: Of the pre-transplant factors studied, OGTT $2 \mathrm{~h}$-CPI proved to be an independent predictor of PTDM.

Keywords: Kidney transplantation; post-transplant diabetes mellitus (PTDM); C-peptide index (CPI); hemoglobin A1c (HbA1c); predictor

Submitted Sep 08, 2019. Accepted for publication Sep 23, 2019.

doi: 10.21037/atm.2019.10.14

View this article at: http://dx.doi.org/10.21037/atm.2019.10.14

\section{Introduction}

Post-transplant diabetes mellitus (PTDM) is a severe and increasingly frequent complication in kidney transplant recipients, which increases the incidence of cardiovascular and infectious complications and has an adverse impact on both graft and patient survival (1-3). PTDM most frequently develops in the early months after transplantation, usually within the first year (2). In kidney transplant recipients, a number of independent risk factors for PTDM have been identified, including older age, obesity, ethnicity, family history of diabetes, donor type, episodes of acute rejection, cytomegalovirus (CMV) and hepatitis $\mathrm{C}$ virus (HCV) infection, the use of corticosteroid, and the type of immunosuppression therapy $(2,4-6)$. Therefore, a pre-transplant identification of patients who are at a high risk of developing PTDM would be beneficial for creating preventive strategies and improving clinical management.

The C-peptide index (CPI) is the ratio of serum $\mathrm{C}$-peptide to plasma glucose levels and is used to evaluate 


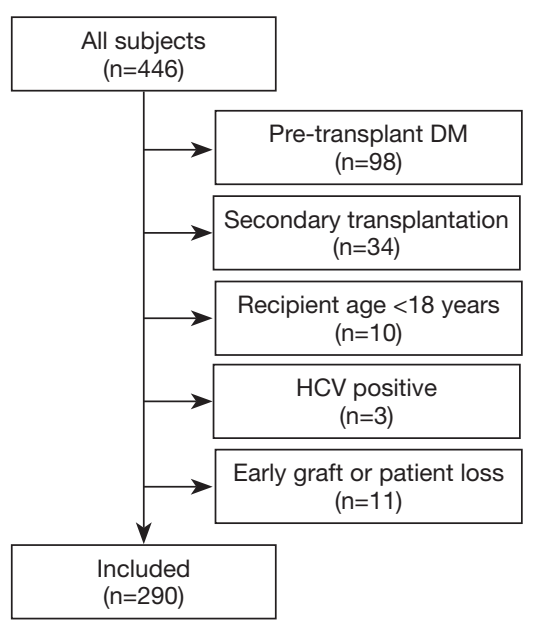

Figure 1 Patient selection pre-transplant.

$\beta$-cell function $(7,8)$. CPI is strongly correlated with $\beta$-cell area as estimated by histological analysis of surgical specimens of the human pancreas (9). It has been reported that postprandial CPI is more practical for evaluating $\beta$-cell functional capacity than fasting CPI (8), and is the best predictive marker for the requirement of insulin therapy (10). Furthermore, studies have demonstrated that postprandial CPI is more related to new onset type 2 diabetes than fasting CPI and the 2-hour glucose test (11). While studies have associated CPI with diabetes, its association with PTDM has not been previously examined.

At our transplant center, all kidney transplant candidates routinely undergo a $75 \mathrm{~g}$ oral glucose tolerance test (OGTT) to identify glucose intolerance or pre-transplant diabetes mellitus. To evaluate the predictive power of CPI for the development of PTDM within one year after transplantation, we retrospectively analyzed 290 kidney transplant recipients with the aim of identifying the time point when OGTT CPI was most closely related to PTDM.

\section{Methods}

\section{Selection of the population}

This retrospective cohort study was approved by the Institutional Review Board/Ethics Committee of The Second Affiliated Hospital of Guangzhou Medical University (approval ID: 2019-hg-ks-01), and all aspects of the study complied with the Helsinki Declaration of 1975. The Ethics Committee of The Second Affiliated Hospital of Guangzhou Medical University specifically approved that no informed consent was required because all data were going to be analyzed anonymously. All of the organs were from donation after citizen's death or from living relative donors, and all of the organ donors had provided informed written consent.

We retrospectively identified 446 consecutive patients receiving kidney transplants in our transplant center between January 2015 and December 2017. A total of 156 patients were excluded from this study for the following reasons: pre-transplant diabetes mellitus $(\mathrm{n}=98)$, secondary transplantation $(n=34)$, recipient age $<18$ years $(n=10)$, or early graft or patient loss $(\mathrm{n}=11)$. HCV positive patients exclusions were due to the low number $(n=3)$. Finally, 290 patients were included in this study (Figure 1). The mean age of the patients was $39.4 \pm 10.2$ years, and the mean pretransplant BMI was $22.1 \pm 3.2 \mathrm{~kg} / \mathrm{m}^{2}$.

\section{Immunosuppression}

Induction therapy consisted of either anti-thymoglobulin (ATG, Fresenius Biotech, Germany), thymoglobulin (Genzyme, Boston, MA, USA), or basiliximab (Simulect, Novartis Pharma, Switzerland).

All patients were treated with corticosteroids, which began with intraoperative $500 \mathrm{mg}$ intravenous (IV) methylprednisolone, followed by 500, 250, and $250 \mathrm{mg}$ IV methylprednisolone on day 1 , day 2 and day 3, respectively, relative to the day of operation. Oral prednisone started with a dose of $20 \mathrm{mg} /$ day on day 4 , with gradual tapering to $8 \mathrm{mg} /$ day at month 6 , and $4 \mathrm{mg} / \mathrm{day}$ thereafter. Corticosteroid reduction was more rapid in patients who developed PTDM.

All patients received $1,000 \mathrm{mg}$ of oral mycophenolate mofetil (MMF) preoperatively, followed by $2 \times 500 \mathrm{mg} /$ day of MMF or $2 \times 360 \mathrm{mg} /$ day of mycophenolate sodium if the patient weighed $\leq 60 \mathrm{~kg}$, or by $2 \times 750 \mathrm{mg} /$ day of $\mathrm{MMF}$ or $2 \times 540 \mathrm{mg} /$ day of mycophenolate sodium if the patient weighed $>60 \mathrm{~kg}$. Calcineurin inhibitor (CNI) therapy was started on the transplant day, using either cyclosporine A (CsA) or tacrolimus (Tac). The CsA dose was adjusted to maintain plasma trough CsA levels of $150-200 \mathrm{ng} / \mathrm{mL}$, while the Tac dose was adjusted to maintain plasma trough FK506 levels of 5-10 ng/mL.

\section{OGTT and laboratory data}

As a screening test, a $75 \mathrm{~g}$ OGTT was performed after an overnight fast of at least $10 \mathrm{~h}$ several months prior to transplantation. Blood samples were obtained before and 
at 1 and $2 \mathrm{~h}$ after ingestion of a $75 \mathrm{~g}$ glucose solution. Biochemical data were retrieved from the laboratory data base, including glycemic profiles (fasting, 1 and $2 \mathrm{~h}$ glucose), C-peptide levels (fasting, 1 and $2 \mathrm{~h}$ ), glycated hemoglobin (HbA1c), albumin, triglycerides, and total cholesterol. Clinical data were gathered from electronic medical records. CPI was calculated as follows: serum C-peptide level $(\mathrm{ng} / \mathrm{mL}) /$ plasma glucose level $(\mathrm{mmol} / \mathrm{L})$.

\section{Definition of PTDM}

In accordance with the American Diabetes Association (ADA) criteria (12), PTDM was diagnosed if one of the following was present during the first year posttransplantation: (I) a fasting plasma glucose (FPG) $\geq 7.0 \mathrm{mmol} / \mathrm{L}$ on two separate occasions, (II) a casual plasma glucose $\geq 11.1 \mathrm{mmol} / \mathrm{L}$ with symptoms of DM, (III) a 2-hour glucose level of OGTT $\geq 11.1 \mathrm{mmol} / \mathrm{L}$, (IV) the need for insulin or oral antidiabetic medication.

\section{Statistical analysis}

Data were analyzed using the SPSS 19.0 software. Descriptive statistics are expressed as mean $\pm \mathrm{SD}$ or median (interquartile range). Distribution of normality was assessed by the Kolmogorov-Smirnov test. Differences between groups were tested by the Student's $t$-test or the non-parametric Mann-Whitney $U$ test as appropriate. Categorical variables were analyzed by the chi-square test.

A logistic regression model was established to assess the independent risk factors associated with PTDM. Covariates of the final model were defined using a backward methodology. A $P$ value $<0.05$ was considered statistically significant.

A survival analysis was carried out to calculate the cumulative probability of PTDM over time. A KaplanMeier analysis was performed to compare survival time (time period without PTDM) according to $2 \mathrm{~h}$-CPI level (1 if $2 \mathrm{~h}$-CPI $\leq 2.60$ and 0 otherwise). A log-rank test was used to compare the obtained survival curves.

A Cox proportional hazard model was constructed to analyze the independent predictors associated with PTDM. Covariates included age, body mass index (BMI), HbA1c, and 2 h-CPI. A P value less than 0.05 was considered statistically significant.

\section{Results}

Of the 290 non-diabetic kidney transplant recipients, 12.4\% $(\mathrm{n}=36)$ developed PTDM within 1 year post-transplantation according to the ADA 2018 criteria (12). The mean time to the development of PTDM was 158 days posttransplantation, and $36.1 \%(13 / 36)$ of patients developed PTDM within 3 months, while $80.6 \%$ developed PTDM (29/36) within 6 months.

No differences were found between PTDM and nonPTDM patients regarding sex, dialysis mode, type of primary disease, or type of CNI. Patients with PTDM had higher pre-transplant age $(\mathrm{P}<0.001)$ and $\mathrm{BMI}(\mathrm{P}=0.004)$ than the non-PTDM patients. A greater proportion of PTDM patients received kidney transplantation from deceased donors compared to non-PTDM patients (97.2\% vs. $85 \%$ respectively, $\mathrm{P}=0.045)$. The patient characteristics of the study are shown in Table 1.

Regarding the biochemical parameters, there were no differences with respect to albumin, triglycerides, and total cholesterol between the two groups, but PTDM patients showed significantly higher HbA1c levels than non-PTDM patients $(\mathrm{P}=0.001)$. No differences were found in fasting CPI and 1h-CPI between the two groups, whereas PTDM patients displayed significantly lower $2 \mathrm{~h}$-CPI levels than non-PTDM patients ( $\mathrm{P}=0.02$, Table 2).

Univariate logistic regression analysis showed that age, pretransplant BMI, primary disease, $\mathrm{HbA1c}$, and $2 \mathrm{~h}-\mathrm{CPI}$ were significant predictors of PTDM (Table 3). In the multiple logistic regression model, variables with a $\mathrm{P}<0.1$ in the univariate analysis were included in analysis (including age, pretransplant $\mathrm{BMI}$, primary disease, type of donation, HbA1c, total cholesterol, and 2 h-CPI). This model showed that age, BMI, HbA1c, and $2 \mathrm{~h}$-CPI were significant pretransplant predictors for PTDM. 2 h-CPI was the only protective factor for PTDM. For every 1-unit increase in $2 \mathrm{~h}$-CPI level, there was a $43.2 \%$ decrease in the risk of developing PTDM (Table 4).

Correlation analysis between $2 \mathrm{~h}$-CPI and other continuous variables was conducted using Spearman's correlation coefficient. A significant direct correlation was found between $2 \mathrm{~h}-\mathrm{CPI}$ and BMI $(\mathrm{r}=0.207, \mathrm{P}<0.001)$, $2 \mathrm{~h}-\mathrm{CPI}$ and type of CNI ( $\mathrm{r}=0.134, \mathrm{P}=0.023), 2 \mathrm{~h}-\mathrm{CPI}$ and HbA1c ( $r=0.154, P=0.009), 2$ h-CPI and albumin $(r=0.219$, $\mathrm{P}<0.001)$, and $2 \mathrm{~h}-\mathrm{CPI}$ and triglycerides $(\mathrm{r}=0.17, \mathrm{P}=0.004)$. There was no correlation between $2 \mathrm{~h}$-CPI and age, and between $2 \mathrm{~h}$-CPI and total cholesterol.

The PH Cox model for the time to PTDM is shown in Table 5. Age, BMI, and HbA1c were introduced as continuous variables. The Cox model revealed that the risk of PTDM increased by about $4 \%$ when age increased by 
Table 1 Patient characteristics

\begin{tabular}{lccc}
\hline Patient characteristics & $\begin{array}{c}\text { Non-PTDM } \\
(\mathrm{N}=254)\end{array}$ & $\begin{array}{c}\text { PTDM } \\
(\mathrm{N}=36)\end{array}$ & P value \\
\hline Age (years) & $38.6 \pm 10$ & $45.2 \pm 10.2$ & $<0.001$ \\
Male recipients, $\mathrm{n}(\%)$ & $158(62.2)$ & $20(55.6)$ & 0.443 \\
Pre-transplant BMI (kg/m²) & $21.9 \pm 3.2$ & $23.5 \pm 3.1$ & 0.004 \\
Dialysis, $\mathrm{n}(\%)$ & & & 0.06 \\
Non-dialysis & $24(9.4)$ & $7(19.4)$ & \\
Hemodialysis & $181(71.3)$ & $19(52.8)$ & \\
Peritoneal dialysis & $49(19.3)$ & $10(27.8)$ & \\
Primary disease, $\mathrm{n}(\%)$ & & & 0.128 \\
Chronic glomerulonephritis & $206(81.1)$ & $26(72.2)$ & \\
lgA nephropathy & $29(11.4)$ & $3(8.3)$ & \\
Polycystic kidney & $9(3.5)$ & $3(8.3)$ & \\
Others & $10(3.9)$ & $4(11.1)$ & \\
Type of donation, $\mathrm{n}(\%)$ & & & \\
Deceased donor & $216(85.0)$ & $35(97.2)$ & \\
Living donor & $38(15.0)$ & $1(2.8)$ & \\
CNI & & & \\
Cyclosporine & & & \\
Tacrolimus & & & \\
\hline
\end{tabular}

PTDM, post-transplant diabetes mellitus; BMI, body mass index; $\mathrm{CNI}$, calcineurin inhibitor. To test for differences between nonPTDM and PTDM patients, categorical variables were analyzed by chi-square test, and quantitative variables were analyzed by Mann-Whitney $U$ tests.

Table 2 Values for pre-transplant biochemical parameters

\begin{tabular}{lccc}
\hline Parameters & Non-PTDM (N=254) & PTDM (N=36) & P value \\
\hline HbA1c (\%) & $5.0 \pm 0.6$ & $5.35 \pm 0.62$ & 0.001 \\
ALB (g/L) & $41.9(37.7-45.2)$ & $41.7(36.3-45.4)$ & 0.725 \\
TG (mmol/L) & $1.31(0.94-1.8)$ & $1.46(0.95-2.00)$ & 0.412 \\
CHOL (mmol/L) & $4.22(3.65-4.97)$ & $4.51(3.60-5.43)$ & 0.259 \\
Fasting-CPI & $1.75(1.29-2.64)$ & $1.75(1.16-2.55)$ & 0.487 \\
1 h-CPI & $1.84(1.46-2.52)$ & $1.75(1.38-2.19)$ & 0.112 \\
2 h-CPI & $2.71(2.52-3.53)$ & $2.19(1.74-2.85)$ & 0.02 \\
\hline
\end{tabular}

PTDM, post-transplant diabetes mellitus; ALB, albumin; TG, triglycerides; CHOL, total cholesterol; CPI, C-peptide index. Values are expressed as mean \pm SD or median (interquartile range), and $\mathrm{P}$ value was obtained using the Student's $t$-test or Mann-Whitney U test.
Table 3 Univariate logistic analysis of factors predicting PTDM

\begin{tabular}{lccc}
\hline Parameters & OR & $95 \% \mathrm{Cl}$ & $\mathrm{P}$ value \\
\hline Age (years) & 1.066 & $1.028-1.104$ & $<0.001$ \\
Sex (male/female) & 0.759 & $0.375-1.537$ & 0.444 \\
Pre-transplant BMI $\left(\mathrm{kg} / \mathrm{m}^{2}\right)$ & 1.164 & $1.048-1.294$ & 0.005 \\
Dialysis mode & 0.951 & $0.503-1.798$ & 0.877 \\
Primary disease & 1.453 & $1.004-2.101$ & 0.047 \\
Type of donation (deceased/ & 0.162 & $0.022-1.221$ & 0.077 \\
living) & & & \\
CNI (Tac/CsA) & 1.262 & $0.581-2.739$ & 0.556 \\
HbA1c (\%) & 2.523 & $1.415-4.5$ & 0.002 \\
ALB (g/L) & 0.999 & $0.946-1.055$ & 0.973 \\
TG (mmol/L) & 1.015 & $0.792-1.302$ & 0.907 \\
CHOL (mmol/L) & 1.299 & $0.989-1.706$ & 0.06 \\
Fasting-CPI & 0.877 & $0.622-1.237$ & 0.455 \\
1 h-CPI & 0.728 & $0.46-1.153$ & 0.176 \\
2 h-CPI & 0.684 & $0.479-0.977$ & 0.037 \\
\hline
\end{tabular}

PTDM, post-transplant diabetes mellitus; OR, odds ratio; $\mathrm{Cl}$, confidence interval; BMI, body mass index; CNI, calcineurin inhibitor; Tac, tacrolimus; CsA, cyclosporine; ALB, albumin; TG, triglycerides; CHOL, total cholesterol; CPI, C-peptide index.

Table 4 Multivariate logistic regression analysis for the study of factors predicting PTDM

\begin{tabular}{lccc}
\hline Parameters & OR & $95 \% \mathrm{Cl}$ & P value \\
\hline Age (years) & 1.04 & $1.001-1.082$ & 0.047 \\
Pre-transplant BMI $\left(\mathrm{kg} / \mathrm{m}^{2}\right)$ & 1.148 & $1.017-1.297$ & 0.026 \\
Primary disease & 1.444 & $0.974-2.142$ & 0.068 \\
$\begin{array}{l}\text { Type of donation (deceased/ } \\
\text { living) }\end{array}$ & 0.281 & $0.035-2.253$ & 0.232 \\
HbA1c (\%) & 2.103 & $1.062-4.162$ & 0.033 \\
CHOL (mmol/L) & 1.066 & $0.79-1.439$ & 0.675 \\
2 h-CPI & 0.568 & $0.379-0.852$ & 0.006 \\
\hline
\end{tabular}

PTDM, post-transplant diabetes mellitus; OR, odds ratio; $\mathrm{Cl}$, confidence interval; $\mathrm{BMI}$, body mass index; $\mathrm{CHOL}$, total cholesterol; CPI, C-peptide index. 
Table 5 The Cox proportional model for the study of the development of PTDM

\begin{tabular}{lccccc}
\hline Parameters & $\beta$ & SE & p-value & Exp $(\beta)$ & $95 \% \mathrm{Cl}$ \\
\hline Age & 0.043 & 0.018 & 0.015 & 1.044 & $1.008-1.080$ \\
BMl & 0.111 & 0.049 & 0.025 & 1.117 & $1.014-1.230$ \\
HbA1c & 0.632 & 0.293 & 0.031 & 1.881 & $1.059-3.341$ \\
2 h-CPI & 1.250 & 0.374 & 0.001 & 3.492 & $1.677-7.268$ \\
\hline
\end{tabular}

PTDM, post-transplant diabetes mellitus; Cl, confidence interval; BMI, body mass index; CPI, C-peptide index.

1 year $[\exp (\beta)=1.044$ with $95 \%$ CI: $1.008,1.080]$. For BMI, the model showed that the risk of PTDM increased by more than $10 \%$ when $\mathrm{BMI}$ increased by 1 unit $[\exp (\beta)=1.117$ with 95\% CI: 1.014, 1.23]. For HbA1c, the Cox model showed that the risk of PTDM increased by near $80 \%$ when HbA1c increased by 1 unit $[\exp (\beta)=1.881$ with $95 \%$ CI: $1.059,3.341]$. Finally, 2 h-CPI was introduced as a categorical variable; patients with lower levels of 2 h-CPI $(<2.60)$ had a risk of PTDM three times higher than patients with 2 h-CPI $>2.60$ $[\exp (\beta)=3.492$ with $95 \%$ CI: $1.677,7.268]$.

Figure 2 shows the Kaplan-Meier survival curve for time to developing PTDM with a comparison of patients according to the age, BMI, HbAlc, and $2 \mathrm{~h}-\mathrm{CPI}$ indicators. According to the age indicator ( 1 if age $\geq 45$ years and 0 if age $<45$ years), patients with older age had a shorter period until PTDM (log-rank =5.248, $\mathrm{P}=0.022$ ). According to the $\mathrm{BMI}$ indicator (1 if $\mathrm{BMI} \geq 25 \mathrm{~kg} / \mathrm{m}^{2}$ and 0 if $\mathrm{BMI}$ $<25 \mathrm{~kg} / \mathrm{m}^{2}$ ), patients with higher BMI had a shorter period until PTDM (log-rank =4.928, $\mathrm{P}=0.026)$. According to the HbA1c indicator ( 1 if $\mathrm{HbA} 1 \mathrm{c} \geq 5.0 \%$ and 0 if $\mathrm{HbAlc}$ $<5.0 \%$ ), patients with higher HbA1c level had a shorter period until PTDM (log-rank =6.957, P=0.008). Finally, according to the $2 \mathrm{~h}-\mathrm{CPI}$ level, with a median cut-off point of 2.60 ( 1 if 2 h-CPI $\leq 2.60$ and 0 if 2 h-CPI >2.60), Patients with lower values of $2 \mathrm{~h}-\mathrm{CPI}$ had a shorter period until PTDM (log-rank =6.299, $\mathrm{P}=0.012$ ).

\section{Discussion}

PTDM is a well-known and long-term complication in kidney transplant patients, with an incidence reports varying from $2 \%$ to $50 \%(13,14)$. The high variability of incidence might be due to the lack of a consensus definition of PTDM, and the differences in population characteristics, criteria for diagnosis, and length of study period (14). In a meta-analysis that integrated various studies, the overall morbidity of PTDM was $21 \%$ (15). Cho et al. (16) reported a $52.4 \%$ cumulative incidence rate of PTDM at 1 month and $57.1 \%$ at 3 to 6 months post-transplant, while Bora et al. (17) found that $61.5 \%$ of patients developed PTDM within the first 3 months and $92.3 \%$ within 6 months post-transplant. In the present study, we observed that 36 patients $(12.4 \%)$ developed PTDM by 1 year posttransplant according to the ADA 2018 criteria (12). Among them, $36.1 \%$ of patients developed PTDM within the first 3 months and $80.6 \%$ within 6 months.

Various factors have been identified as risk factors for the development of PTDM, including age, obesity, race, family history of DM, acute rejection, chronic infection with $\mathrm{HCV}$, and the type of immunosuppression used (4-6). Our study supports previous results showing that older age is a risk factor for the development of PTDM, as the risk of PTDM increases by about $4 \%$ when age increases by one year. Moreover, patients who are older than 45 years show a greater cumulative probability of PTDM occurrence compared to that of younger patients. Casio and colleagues have reported that the risk of developing PTDM increases in kidney transplant recipients older than 40 years (13). This is not surprising as older age is a risk factor for the development of diabetes in the non-transplant population as well.

Obesity is a well-known risk factor that contributes to the development of PTDM (18). In a meta-analysis including 55 eligible studies, BMI was found to be an independent risk factor for PTDM (15). In this study, pre-transplant $\mathrm{BMI}$ was an independent predictor for the development of PTDM in kidney transplant recipients. Patients who have developed PTDM present a higher pretransplant BMI, which is in line with previous results. In fact, obesity is associated with hypertriglyceridaemia, lowgrade inflammation, and decreased adiponectin release, all of which induce insulin resistance (19). Furthermore, our results also show that patients who are overweight (BMI $\geq 25 \mathrm{~kg} / \mathrm{m}^{2}$ ) show a greater cumulative probability of PTDM than those of normal weight. Our findings also indicate that for each 1-unit increase in pre-transplant BMI, the risk of developing PTDM increases by more than $10 \%$. 

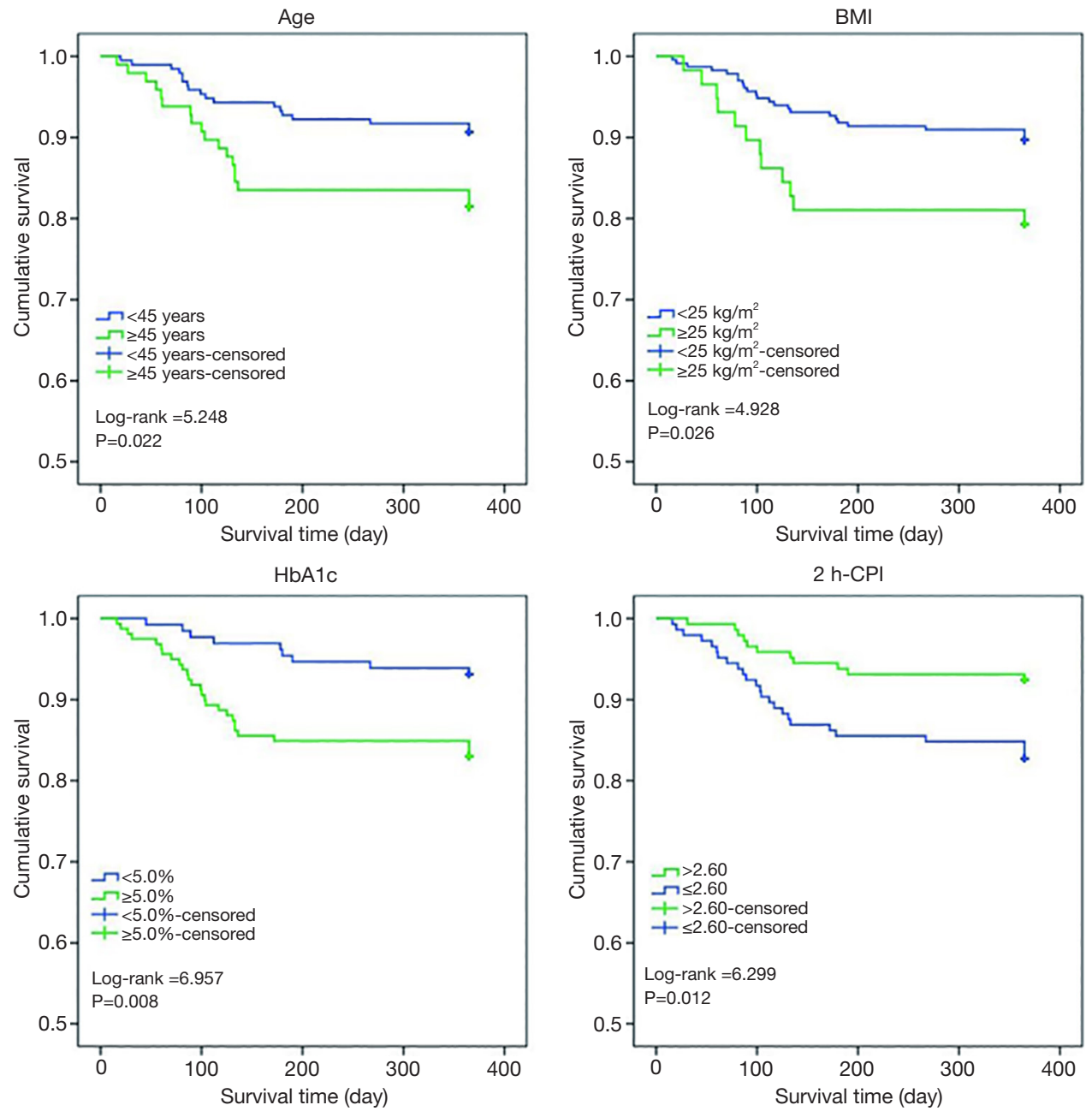

Figure 2 Kaplan-Meier curve estimating the development of PTDM in relation to pre-transplant age, BMI, HbAlc, and 2 h-CPI. BMI, body mass index; CPI, C-peptide index.

HbA1c is a validated and reliable marker for glycemic control (20). According to the ADA, an HbA1c level $\geq 6.5 \%$ has been included as a criterion for the diagnosis of diabetes mellitus (21). In diabetic patients, HbA1c is an important predictor of morbidity and mortality (22). In the general population, even having HbA1c levels $<6.5 \%$ may indicate a risk of developing diabetes mellitus (23). Erhan's study shows that pre-transplant $\mathrm{HbAlc}$ is a predictive risk marker for early diagnosis of PTDM in kidney transplant patients (24). In this study, our results suggest that HbAlc is a predictor of PTDM, and the risk of PTDM increases as HbA1c levels increase.

The CPI has recently been devised as an index of $\beta$-cell function $(8,25)$, which is often measured as fasting CPI or postprandial CPI. Previous studies have shown the clinical usefulness of the CPI, as CPI is strongly correlated with $\beta$-cell area as assessed by histological analysis of surgical specimens of the pancreas $(7,9)$, and decreases progressively with the duration of type 2 diabetes (26). Moreover, postprandial CPI is more strongly correlated with the insulin requirement for the treatment of type $2 \mathrm{DM}$ compared with fasting CPI (8). Based on OGTT, Yoko and colleagues found that $2 \mathrm{~h}$-CPI was significantly correlated with both the oral disposition and clamp disposition index, which are two other indices used for evaluating insulin secretion, suggesting that OGTT $2 \mathrm{~h}-\mathrm{CPI}$ is related to both insulin secretion and insulin sensitivity (27). Another recent study demonstrated that postprandial CPI is more related to diabetes than fasting CPI, and OGTT $1 \mathrm{~h}-\mathrm{CPI}$ is most related to diabetes in patients without diabetes (28). 
However, in this study, we did not find any relationship between OGTT 1h-CPI and PTDM; instead, OGTT $2 \mathrm{~h}$-CPI was an independent predictor of PTDM. For every unit decrease in $2 \mathrm{~h}$-CPI, there was a $43.2 \%$ increase in the risk of developing PTDM. In the survival analysis, patients with low pre-transplant $2 \mathrm{~h}$-CPI levels had a higher probability of developing PTDM.

In conclusion, the results suggest that, in the pretransplant stage, age, BMI, HbAlc, and OGTT 2 h-CPI are independent predictors of PTDM in kidney transplantation. A higher 2 h-CPI level constitutes a protective factor against PTDM in kidney transplant patients. We believe that post-transplant management and immunosuppression should be individualized depending on these risk factors.

\section{Acknowledgments}

Funding: This work was supported by the Youth Scholar project of National Natural Science Foundation of China (grant number 81601393); the Scientific Research Project of the Higher Education Institutions of Guangzhou, China (grant number 1201630506).

\section{Footnote}

Conflicts of Interest: The authors have no conflicts of interest to declare.

Ethical Statement: The authors are accountable for all aspects of the work in ensuring that questions related to the accuracy or integrity of any part of the work are appropriately investigated and resolved. This retrospective cohort study was approved by the Institutional Review Board/Ethics Committee of The Second Affiliated Hospital of Guangzhou Medical University (approval ID: 2019-hgks-01), and all aspects of the study complied with the Helsinki Declaration of 1975. The Ethics Committee of The Second Affiliated Hospital of Guangzhou Medical University specifically approved that no informed consent was required because all data were going to be analyzed anonymously.

\section{References}

1. Miles AM, Sumrani N, Horowitz R, et al. Diabetes mellitus after renal transplantation: as deleterious as non-transplant-associated diabetes? Transplantation 1998;65:380-4.
2. Kasiske BL, Snyder JJ, Gilbertson D, et al. Diabetes mellitus after kidney transplantation in the United States. Am J Transplant 2003;3:178-85.

3. Moreau K. Post-transplantation diabetes mellitus. Nephrol Ther 2006;2 Suppl 1:S71-6.

4. Heisel O, Heisel R, Balshaw R, et al. New onset diabetes mellitus in patients receiving calcineurin inhibitors: a systematic review and meta-analysis. Am J Transplant 2004;4:583-95.

5. Araki M, Flechner SM, Ismail HR, et al. Posttransplant diabetes mellitus in kidney transplant recipients receiving calcineurin or mTOR inhibitor drugs. Transplantation 2006;81:335-41.

6. Sharif A, Baboolal K. Risk factors for new-onset diabetes after kidney transplantation. Nat Rev Nephrol 2010;6:415-23.

7. Meier JJ, Menge BA, Breuer TG, et al. Functional assessment of pancreatic beta-cell area in humans. Diabetes 2009;58:1595-603.

8. Saisho Y, Kou K, Tanaka K, et al. Postprandial serum $\mathrm{C}$-peptide to plasma glucose ratio as a predictor of subsequent insulin treatment in patients with type 2 diabetes. Endocr J 2011;58:315-22.

9. Meier JJ, Breuer TG, Bonadonna RC, et al. Pancreatic diabetes manifests when beta cell area declines by approximately $65 \%$ in humans. Diabetologia 2012;55:1346-54.

10. Saisho Y. Postprandial C-Peptide to Glucose Ratio as a Marker of beta Cell Function: Implication for the Management of Type 2 Diabetes. Int J Mol Sci 2016;17. doi: 10.3390/ijms17050744.

11. Kim JD, Kang SJ, Lee MK, et al. C-Peptide-Based Index Is More Related to Incident Type 2 Diabetes in NonDiabetic Subjects than Insulin-Based Index. Endocrinol Metab (Seoul) 2016;31:320-7.

12. Classification and Diagnosis of Diabetes: Standards of Medical Care in Diabetes-2018. Diabetes Care 2018;41:S13-27.

13. Cosio FG, Pesavento TE, Osei K, et al. Post-transplant diabetes mellitus: increasing incidence in renal allograft recipients transplanted in recent years. Kidney Int 2001;59:732-7.

14. Montori VM, Basu A, Erwin PJ, et al. Posttransplantation diabetes: a systematic review of the literature. Diabetes Care 2002;25:583-92.

15. Chang S, Jiang J. Association of Body Mass Index and the Risk of New-Onset Diabetes After Kidney Transplantation: A Meta-analysis. Transplant Proc 2018;50:1316-25. 
16. Cho YM, Park KS, Jung HS, et al. High incidence of tacrolimus-associated posttransplantation diabetes in the Korean renal allograft recipients according to American Diabetes Association criteria. Diabetes Care 2003;26:1123-8.

17. Bora GS, Guleria S, Reddy VS, et al. Risk factors for the development of new-onset diabetes mellitus in a living related renal transplant program. Transplant Proc 2010;42:4072-3.

18. Jenssen T, Hartmann A. Post-transplant diabetes mellitus in patients with solid organ transplants. Nat Rev Endocrinol 2019;15:172-88.

19. Després JP, Lemieux I. Abdominal obesity and metabolic syndrome. Nature 2006;444:881-7.

20. Dunn PJ, Cole RA, Soeldner JS, et al. Reproducibility of hemoglobin AIc and sensitivity to various degrees of glucose intolerance. Ann Intern Med 1979;91:390-6.

21. International-Expert-Committee. International Expert Committee report on the role of the A1C assay in the diagnosis of diabetes. Diabetes Care 2009;32:1327-34.

22. Ishimura E, Okuno S, Kono K, et al. Glycemic control and survival of diabetic hemodialysis patients--importance of lower hemoglobin A1C levels. Diabetes Res Clin Pract 2009;83:320-6.

23. Ginde AA, Cagliero E, Nathan DM, et al. Value of risk

Cite this article as: Lai X, Zhang L, Fang J, Li G, Xu L, Ma J, Xiong Y, Liu L, Chen Z. OGTT 2-hour serum C-peptide index as a predictor of post-transplant diabetes mellitus in kidney transplant recipients. Ann Transl Med 2019;7(20):538. doi: 10.21037/atm.2019.10.14 stratification to increase the predictive validity of $\mathrm{HbA1c}$ in screening for undiagnosed diabetes in the US population. J Gen Intern Med 2008;23:1346-53.

24. Tatar E, Kircelli F, Demirci MS, et al. Pre-transplant $\mathrm{HbA1c}$ level as an early marker for new-onset diabetes after renal transplantation. Int Urol Nephrol 2013;45:251-8.

25. Funakoshi S, Fujimoto S, Hamasaki A, et al. Utility of indices using $\mathrm{C}$-peptide levels for indication of insulin therapy to achieve good glycemic control in Japanese patients with type 2 diabetes. J Diabetes Investig 2011;2:297-303.

26. Nybäck-Nakell A, Bergstrom J, Adamson U, et al. Decreasing postprandial C-peptide levels over time are not associated with long-term use of sulphonylurea: an observational study. Diabetes Metab 2010;36:375-80.

27. Okuno Y, Komada H, Sakaguchi K, et al. Postprandial serum C-peptide to plasma glucose concentration ratio correlates with oral glucose tolerance test- and glucose clamp-based disposition indexes. Metabolism 2013;62:1470-6.

28. Zhang H, Bian B, Hu F, et al. OGTT 1 h serum C-peptide to plasma glucose concentration ratio is more related to beta cell function and diabetes mellitus. Oncotarget 2017;8:51786-91. 